\title{
Direct innominate artery cannulation: An alternate technique for antegrade cerebral perfusion during aortic hemiarch reconstruction
}

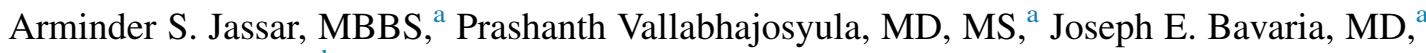 \\ Jacob Gutsche, MD, ${ }^{b}$ Nimesh D. Desai, MD, PhD, ${ }^{a}$ Matthew L. Williams, MD, ${ }^{a}$ \\ Rita K. Milewski, MD, PhD, ${ }^{\mathrm{a}}$ W. Clark Hargrove, $\mathrm{MD},{ }^{\mathrm{a}}$ and Wilson Y. Szeto, $\mathrm{MD}^{\mathrm{a}}$
}

\begin{abstract}
Objective: We describe an alternate technique for establishing antegrade cerebral perfusion (ACP) during hypothermic circulatory arrest via direct, central cannulation of the innominate artery.

Methods: From 2009 to 2015, 100 elective hemiarch reconstructions for proximal aortic aneurysms were performed under moderate hypothermic circulatory arrest (MHCA). Cerebral perfusion was instituted with ACP via direct cannulation of the innominate artery.
\end{abstract}

Results: Mean patient age was $63 \pm 13$ years $(72$ men; $72 \%$ ). Mean MHCA temperature was $27.3^{\circ} \mathrm{C} \pm 1.0^{\circ} \mathrm{C}$ (median, $28^{\circ} \mathrm{C}$ ). Mean $\mathrm{ACP}$ time was $17 \pm 4$ minutes and mean crossclamp time was $134 \pm 42$ minutes. Proximal reconstruction included root replacement with composite valved graft $(n=47)$, valve sparing root reimplantation $(\mathrm{n}=16)$, and aortic valve replacement $(\mathrm{n}=19)$. In-hospital 30-day mortality $(\mathrm{n}=1 ; 1 \%)$, stroke $(1 ; 1 \%)$, reversible ischemic neurologic deficit $(\mathrm{n}=1 ; 1 \%)$, coma $(\mathrm{n}=0)$, and renal failure $(\mathrm{n}=1 ; 1 \%)$ rates were low. There was no incidence of injury or dissection of the innominate artery.

Conclusions: Direct, central innominate artery cannulation for ACP yields excellent outcomes. This technique is safe, provides excellent cerebral protection during circulatory arrest and simplifies the circulatory management strategy for elective ascending aortic and hemiarch reconstruction. ( $\mathrm{J}$ Thorac Cardiovasc Surg 2016;151:1073-8)

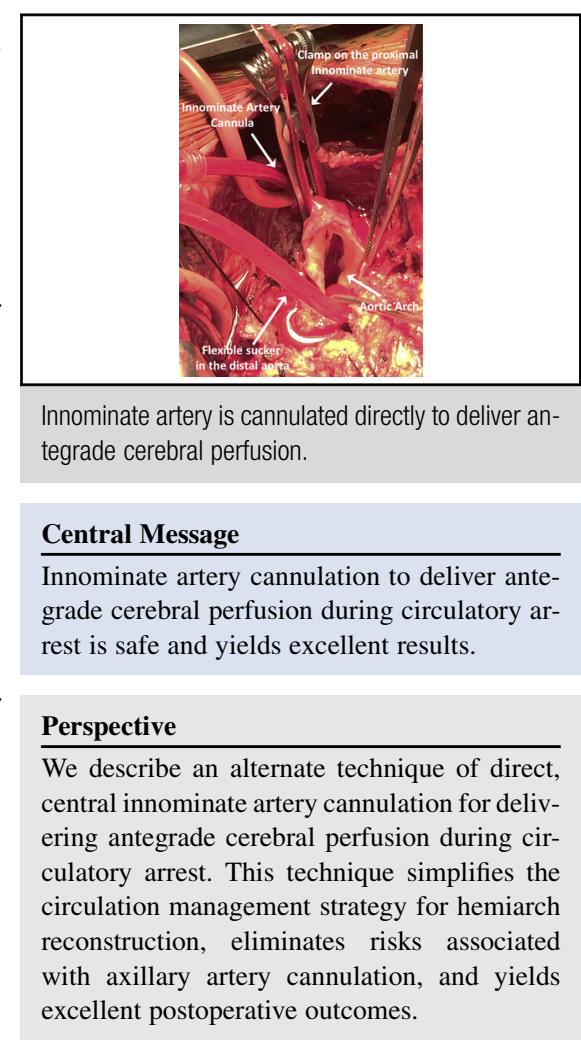

See Editorial Commentary page 1079.
Aortic arch reconstruction requires complex circulatory management and several strategies for cerebral protection during circulatory arrest have been described. These include hypothermia, either alone or in conjunction with either retrograde cerebral perfusion (RCP) or antegrade

\footnotetext{
From the ${ }^{a}$ Division of Cardiovascular Surgery and ${ }^{\mathrm{b}}$ Department of Anesthesia, University of Pennsylvania School of Medicine, Philadelphia, $\mathrm{Pa}$.

A.S.J., P.V., and W.Y.Z. contributed equally to this work.

Supported by the Division of Cardiovascular Surgery, Department of Surgery, University of Pennsylvania, Philadelphia, PA.

Received for publication Aug 6, 2015; revisions received Nov 8, 2015; accepted for publication Nov 17, 2015; available ahead of print Dec 23, 2015.

Address for reprints: Wilson Y. Szeto, MD, Division of Cardiovascular Surgery, University of Pennsylvania, Penn Presbyterian Medical Center, 51 N 39th St, Heart and Vascular Pavilion Suite 2A, Philadelphia, PA 19104 (E-mail: Wilson.Szeto@ uphs.upenn.edu).

$0022-5223 / \$ 36.00$

Copyright (c) 2016 by The American Association for Thoracic Surgery

http://dx.doi.org/10.1016/j.jtcvs.2015.11.027
}

cerebral perfusion (ACP). Although the relative superiority of one technique over the other is still debated, ${ }^{1,2}$ several recent reports have established the safety and efficacy of moderate hypothermia and selective ACP during circulatory arrest for aortic arch reconstruction. ${ }^{3-6}$

ACP is frequently established via axillary artery cannulation through a separate right subclavicular incision. Although this technique is widely used and is generally safe, complication rates of up to $14 \%$ have been reported with axillary artery cannulation. ${ }^{7}$ Recently, the innominate artery has been used as an alternate site for aortic cannulation during aortic

Scanning this QR code will take you to the article title page.

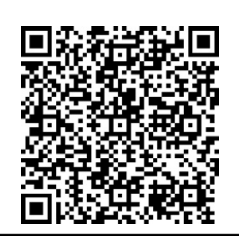




\section{Abbreviations and Acronyms \\ $\mathrm{ACP}=$ antegrade cerebral perfusion \\ $\mathrm{CPB}=$ cardiopulmonary bypass \\ $\mathrm{EEG}=$ electroencephalogram \\ $\mathrm{RCP}=$ retrograde cerebral perfusion}

reconstruction. ${ }^{8-13}$ Another technique of providing ACP is via positioning balloon-tipped catheters into the ostia of the supraaortic vessels, but this can be cumbersome and interfere in the surgical field. We have adopted an alternate technique with direct cannulation of the innominate artery for providing selective ACP during circulatory arrest. In this report, we describe our technique and present our outcomes in patients undergoing elective aortic hemiarch reconstruction using this circulatory arrest management strategy.

\section{METHODS \\ Patients}

Since May 2009, we have performed 100 elective hemiarch reconstructions for aortic aneurysmal disease in which ACP was established via direct cannulation of the innominate artery. For these patients, preoperative, intraoperative, and postoperative characteristics were obtained from the prospectively maintained University of Pennsylvania aortic surgery database and supplemented by surgeons' report of the operation and hospital records. Patients undergoing reconstruction urgently or emergently, or due to endocarditis or aortic dissection, were excluded from this report. The study protocol was approved by the institutional review board of the University of Pennsylvania.

\section{Intraoperative Monitoring}

In addition to standard anesthesia monitors, an oximetric Swan-Ganz catheter and right radial artery catheter are placed for continuous blood pressure and hemodynamic monitoring. Electrodes are placed for continuous intraoperative electroencephalogram (EEG) monitoring by a dedicated technician under the supervision of a staff neurologist to detect cerebral ischemia. In addition, cerebral perfusion is monitored using near-infrared spectroscopy (Somanetics; Covidien, Mansfield, Mass). Baseline cerebral oximetry values are noted and trends monitored throughout the case. Alerts for possible cerebral ischemia are triggered by a $50 \%$ decrease in amplitude in cerebral oximetry values or lateral asymmetry in the EEG waveforms.

\section{Operative Technique}

All patients underwent a full sternotomy for exposure. The brachiocephalic vein is retracted cephalad to expose the aortic arch. The proximal innominate artery is exposed and an umbilical tape is passed around it to allow caudal retraction. Arterial cannulation is established via the ascending aorta (in the aneurysmal portion of the aorta that will eventually be resected). Venous cannulation is established centrally. Aortic crossclamp is applied and the patient is cooled to between $24^{\circ} \mathrm{C}$ and $28^{\circ} \mathrm{C}$ (mean, $27.3^{\circ} \mathrm{C} \pm 1.0^{\circ} \mathrm{C}$; median, $28^{\circ} \mathrm{C}$ ). The initial few patients were cooled to between $24^{\circ} \mathrm{C}$ and $26^{\circ} \mathrm{C}$, but in more recent experience, we cool to $28^{\circ} \mathrm{C}$ systemic temperature.

Proximal aortic reconstruction is performed while the patient is being cooled. Once the target systemic temperature is achieved, a pledgeted 4-0 polypropylene horizontal mattress stitch is placed in the proximal innominate artery and a short tipped 9-Fr cardioplegia cannula (Medtronic, Minneapolis, Minn) (Figure 1) is inserted into the innominate artery just before initiating circulatory arrest (Figure 1). Cardiopulmonary bypass

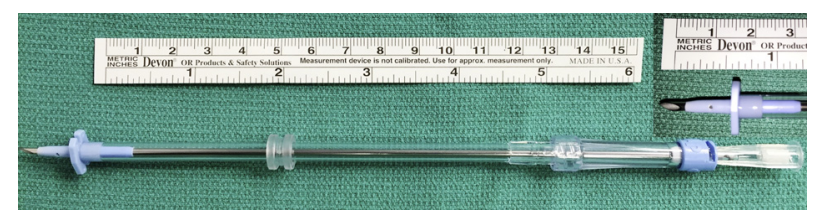

FIGURE 1. Nine-French short-tipped cannula used for cannulating the innominate artery for antegrade cerebral perfusion.

(CPB) is halted to initiate circulatory arrest, and ACP is begun via the innominate artery cannula, with almost no flow interruption to the brain. The proximal innominate artery is occluded using a metal bulldog clamp (Figure 2). Left carotid and left subclavian arteries are not routinely clamped. A flexible pump suction is placed in the distal aorta to maintain a bloodless field. ACP is administered at $24^{\circ} \mathrm{C}$; flow rates are maintained at 10 to $12 \mathrm{~mL} / \mathrm{kg} / \mathrm{min}$ to maintain a right radial artery pressure of 50 to $60 \mathrm{~mm} \mathrm{Hg}$. Transverse hemiarch anastomosis is performed under circulatory arrest in the standard fashion. The polyethylene terephthalate graft is cannulated to resume CPB (Figure 3). At this time, systemic blood pressure is transiently lowered to $\leq 40 \mathrm{~mm} \mathrm{Hg}$, the innominate artery cannula is removed, and the previously placed pledgeted mattress suture is tied down to achieve hemostasis. The remainder of the aortic reconstruction is performed in the standard fashion.

\section{RESULTS}

Preoperative patient characteristics are presented in Table 1. All patients underwent ascending aortic and aortic hemiarch replacement with a prosthetic graft. Details of the proximal reconstruction, concomitant procedures, and operative times are presented in Table 2. Perioperative outcomes are presented in Table 3. There were no dissections or hematoma associated with cannulation of the innominate artery.

The single mortality in this series was an 82-year-old man with critical aortic stenosis, heavily calcified aortic root, and ascending aortic aneurysm. The patient underwent aortic root replacement and transverse hemiarch procedure. Postoperatively, the patient developed worsening right ventricular failure that lead to multisystem organ failure and death.

Two patients ( $2 \%)$ had postoperative neurologic events, neither of which resulted in permanent impairment. One patient experienced a right frontal ischemic stroke that resulted in left upper extremity weakness. This patient recovered full strength in the left arm by the time of his follow-up visit. A second patient experienced a reversible ischemic neurologic deficit with a nonfocal neurologic examination with altered mental status and delirium without evidence of stroke on computed tomography scan. The patient improved over the next few days, without any residual clinical neurologic impairment.

\section{DISCUSSION}

As experience with aortic arch surgery accumulates, cerebral protection strategies during circulatory arrest continue to evolve. The need for establishing deep hypothermia, either alone or in combination with RCP has been obviated by the strategy of ACP under moderate hypothermia. This approach has the potential of 

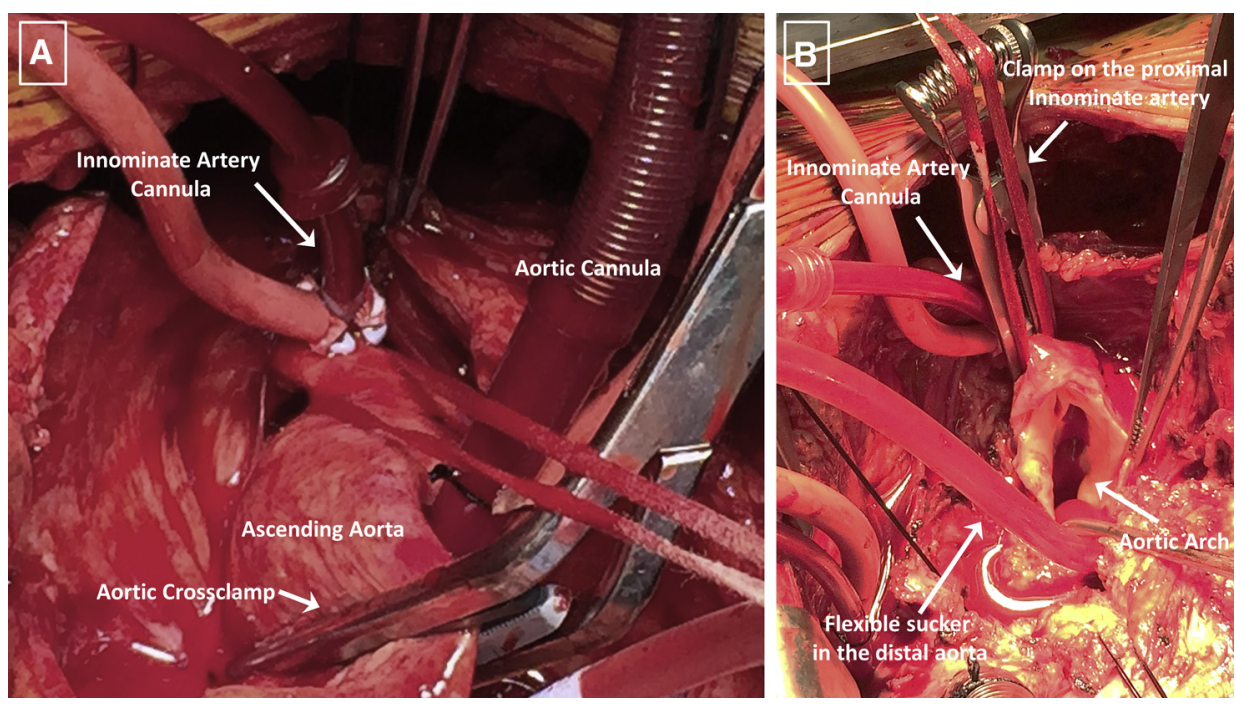

FIGURE 2. A, Innominate artery is circumferentially encircled with an umbilical tape and retracted caudally and a 9-Fr short-tipped cannula is inserted into the innominate artery. B, Under circulatory arrest, proximal aorta is resected, including the site of the aortic cannula. Proximal innominate artery is clamped and antegrade cerebral perfusion is delivered via the 9-Fr cannula. Note that the innominate artery cannula is very low profile and causes no obstruction of the surgical field.

decreasing CPB time by decreasing the time to achieve deep hypothermia and subsequent rewarming. ${ }^{6}$ ACP is frequently established via the right axillary artery, either by direct cannulation or through a side graft sewn to the artery. Although this technique is generally safe and has the advantage of uninterrupted cerebral blood flow, it requires a separate incision and can be associated with complications, including brachial plexus injury/neuropraxia, hematoma formation, and axillary artery injury resulting in limb malperfusion. ${ }^{7,9}$ Another technique for establishing ACP is via placing flexible balloon-tipped catheters into the ostia of 1 or more arch vessels under direct vision once circulatory arrest has been initiated and the aortic arch has been opened. Although this technique avoids the extra incision required for axillary artery exposure and cannulation, it mandates interruption of cerebral blood flow. Additionally, the ostial catheters traverse the surgical field, can be cumbersome, and can get dislodged requiring repositioning and thus waste precious time during circulatory arrest.

Innominate artery cannulation has recently been described as an alternate cannulation site during aortic arch surgery. Two different techniques have been described. Anastasiadis and colleagues ${ }^{8}$ described their technique of direct innominate artery cannulation in 22 patients with a specially designed aortic cannula that is used for both
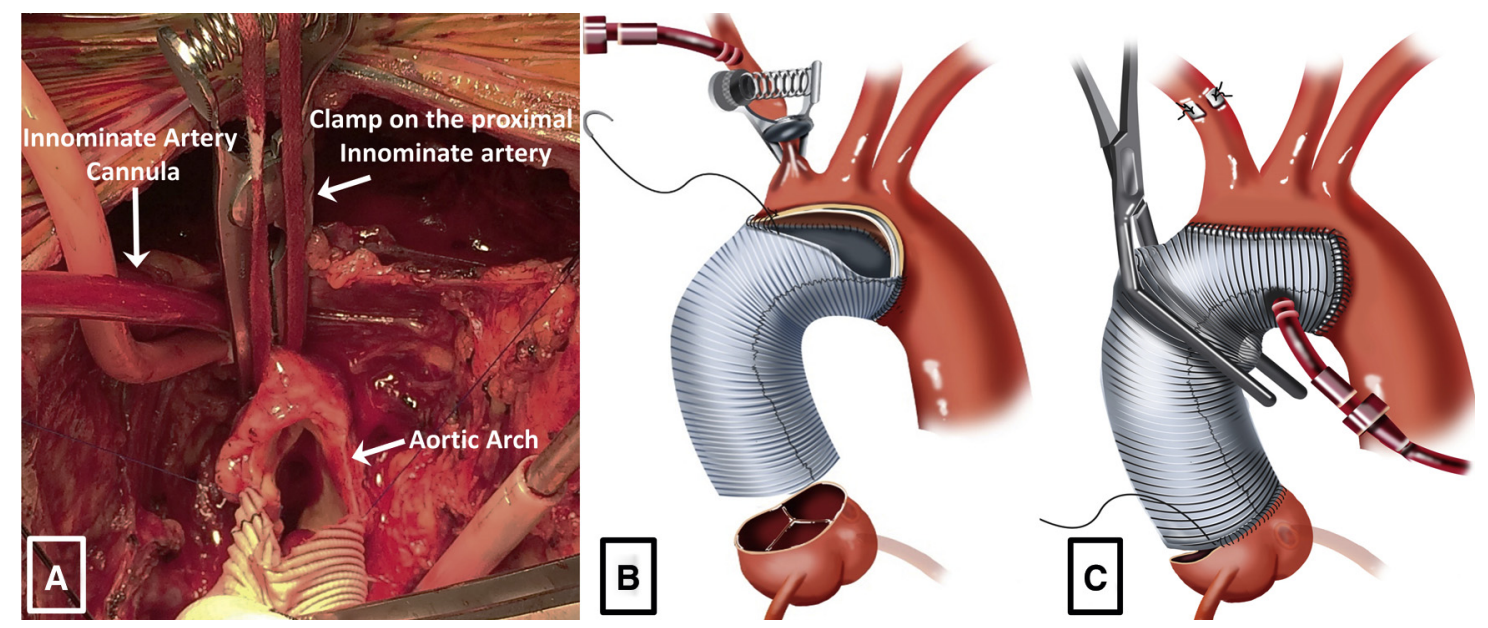

FIGURE 3. A and B, The distal aortic anastomosis is performed with a running suture while antegrade cerebral perfusion is delivered via the innominate artery. C, After distal aortic anastomosis is complete, the innominate artery cannula is removed and the aortic graft is cannulated to re-establish cardiopulmonary bypass. Proximal reconstruction is completed. 
TABLE 1. Preoperative patient characteristics $(N=100)$

\begin{tabular}{lc}
\hline Patient characteristic & Result \\
\hline Age (y) & $63 \pm 13$ \\
Male & 72 \\
Smoker & 25 \\
Hypertension & 66 \\
Diabetes mellitus & 13 \\
Dyslipidemia & 60 \\
Renal failure & 3 \\
Renal failure with dialysis & 0 \\
Chronic lung disease & 10 \\
Peripheral artery disease & 4 \\
Bicuspid aortic valve & 35 \\
Cerebrovascular disease & 12 \\
Cerebrovascular accident & 3 \\
Prior myocardial infarction & 5 \\
Arrhythmia & 10 \\
Redo cardiac surgery & 10 \\
Ejection fraction (\%) & \\
$\quad \leq 30$ & 3 \\
31-50 & 14 \\
>50 & 83 \\
Heart failure & \\
NYHA I & 5 \\
NYHA II & 27 \\
NYHA III & 12 \\
NYHA IV & 1 \\
\hline Vara & \\
\hline
\end{tabular}

Values are presented as $\mathrm{n}$ or mean \pm standard deviation. NYHA, New York Heart Association.

CPB and ACP. Although, detailed perioperative outcomes were not reported, they did not have any postoperative neurologic events associated with this strategy. Other groups have used a side graft sewn to the innominate artery to establish CPB. Di Eusanio and colleagues ${ }^{14}$ reported outcomes in a series of 55 patients undergoing aortic replacement mainly for aneurysmal disease, where the innominate artery was partially clamped and a side graft was attached to the innominate artery 4 to $5 \mathrm{~cm}$ from its origin and used for both CPB and ACP. Hospital mortality was $3.6 \%$, transient neurologic dysfunction rate was $1.8 \%$, and stroke rate was zero. Preventza and colleagues ${ }^{11}$

TABLE 2. Operative procedures and times

\begin{tabular}{lc}
\hline \multicolumn{1}{c}{ Surgical procedures $(\mathbf{N}=\mathbf{1 0 0})$} & Result \\
\hline Ascending aorta/hemiarch replacement & 100 \\
Aortic root replacement with composite valve graft & 47 \\
Aortic root replacement-valve sparing* & 16 \\
Aortic valve replacement & 19 \\
Coronary artery bypass & 20 \\
Mitral valve repair & 6 \\
Operative times & $170 \pm 45$ \\
Cardiopulmonary bypass (min) & $134 \pm 42$ \\
Aortic crossclamp (min) & $17 \pm 4$ \\
Circulatory arrest (min) & \\
Values are presented as n or mean \pm standard deviation. *For example, David V.
\end{tabular}

TABLE 3. Perioperative outcomes

\begin{tabular}{lc}
\hline \multicolumn{1}{c}{ Perioperative outcomes $(\mathbf{N}=\mathbf{1 0 0})$} & Result \\
\hline In-hospital and/or 30-d mortality & 1 \\
Neurologic outcomes & 1 \\
Permanent stroke & 0 \\
Transient ischemic attack & 1 \\
Reversible ischemic neurologic deficit & 0 \\
$\quad$ Paralysis & 1 \\
Renal failure requiring dialysis & 3 \\
Permanent pacemaker & 7 \\
Reoperation for bleeding & 1 \\
Deep sternal infection & 1 \\
Multisystem organ failure & 12 \\
Ventilated $>$ 24 h & 0 \\
Tracheostomy & $7.9 \pm 3.3(7)$ \\
Postoperative hospital length of stay (d) &
\end{tabular}

reported a series of 68 patients undergoing proximal aortic surgery (43 arch aneurysms, aortic dissection in 11 patients, total arch aneurysm in 10 patients, and ascending aortic aneurysm in 4 patients) where a side graft was sewn to the innominate artery either using a partial occlusion clamp or in cases where the size of the innominate artery was not big enough, with complete flow occlusion of the innominate artery using 2 vascular clamps. The graft was used for both CPB and ACP. Thirty-day mortality was $1.5 \%, 3$ patients $(4.4 \%)$ experienced a stroke, 2 patients had a partial recovery, and 7 patients developed postoperative confusion that resolved in all cases. These authors confirmed their excellent results using their technique in a larger series of 263 patients who underwent proximal aortic reconstruction and reported perioperative mortality and permanent stroke rate of $4.9 \%$ and $1.9 \%$, respectively. ${ }^{15}$ Urbanski and colleagues ${ }^{16}$ used moderate hypothermia and ACP during circulatory arrest in 347 patients who underwent nonemergent aortic arch surgery. Arterial cannulation was established via a branch graft anastomosed to either the carotid $(n=320)$ or the innominate artery $(n=19)$. Stroke rate was $0.9 \%$ and temporary postoperative neurologic dysfunction was noted in $2.3 \%$ of patients. In the majority of these cases ( 315 out of 347), access to these vessels was obtained through a separate incision in the neck. This technique of sewing a graft to the innominate artery has all the advantages of axillary artery cannulation; namely, single arterial cannulation for the entire case without any cerebral flow interruption, and also obviates the need for an extra incision. However, it requires extensive dissection of the innominate artery, and either partial or complete arterial occlusion to sew the graft.

Our technique is unique in that we use the innominate artery cannula exclusively for ACP, whereas CPB is established centrally via a cannula placed in the ascending aorta. A short-tipped 9-Fr catheter in the innominate artery is 
adequate for running flows up to $1.5 \mathrm{~L} / \mathrm{min}$, which are necessary for providing ACP. Also, a small cannula is intuitively less traumatic to the innominate artery than sewing a side graft, or even compared with inserting a large cannula to obtain flows that are required for providing full CPB. Our technique requires only minimal dissection of the innominate artery-very similar to what is required to clamp the innominate artery during ACP via axillary or innominate artery. Also, because we do not use the innominate cannula for $\mathrm{CPB}$, the cannula does not need to be redirected toward the aorta or the brain when switching from $\mathrm{CPB}$ to ACP during circulatory arrest, as has been described in some previous reports. ${ }^{12}$ Although the brachiocephalic vein could probably be ligated safely in most cases, if necessary, to expose the innominate artery, this was not required in any patient in this series, including patients undergoing redo sternotomy.

Although our technique involves 3 steps for providing $\mathrm{CPB}$ and $\mathrm{ACP}$, namely cannulating the ascending aorta, cannulating the innominate artery, and finally cannulating the graft, it is still very fast and easy to adopt. Cannulating the aorta or the graft are simple steps that are routinely performed by all cardiac surgeons, and cannulating the innominate artery requires only 1 additional stitch. Our technique requires minimal interruption of cerebral arterial flow compared with that needed to insert ostial catheters for ACP. Unlike the ostial catheters, the small innominate artery cannula has a very low profile, and is positioned away from the operative field (Figure 2). Also, unlike the ostial catheters that frequently get dislodged during the case, our technique ensures a very stable system for providing uninterrupted ACP. The entire operation is accomplished through the standard sternotomy incision without the need for a second incision. Thus the need for a separate axillary artery exposure and the associated risks are eliminated. Additionally, our technique allows reliable monitoring of systemic blood pressure during CPB and cerebral perfusion pressure during cardiac arrest via a catheter placed in the right radial artery. Our results show that this technique is safe, does not result in innominate artery injury or dissection, and can be performed with minimal risk of perioperative mortality and neurologic complications.

In the only other published report with a similar of separate innominate cannulation exclusively for ACP, Garg and colleagues ${ }^{13}$ reported a stroke rate of $2 \%$ in a series of 50 patients undergoing elective ascending aorta or arch replacement. These authors used the Seldinger technique to place a 14-Fr catheter in the innominate artery over a guidewire using serial dilators. The innominate artery could not be cannulated in 2 cases due to the tortuosity in the course of the innominate artery. Our technique of using the smaller (9-Fr) short-tipped cardioplegia cannula obviates the need for passing wires and dilators upstream into the innominate artery, which may further reduce the risk of cerebral atheroembolism. Additionally, our technique can be used even in tortuous vessels because only the cannula tip needs to be positioned in the innominate artery. ${ }^{17}$ To date, there have been no aborted attempts at establishing $\mathrm{ACP}$ in any patient with this technique. We carefully review the preoperative computed tomography scan with particular attention to the degree of calcification in the ascending aorta and the innominate artery. Patients with significant proximal innominate artery calcification may not be candidates for this approach. Additionally, we do not employ this approach for patients with ascending aortic dissections that extend into the innominate artery as catheter positioning into the true arterial lumen cannot be ensured. Our approach in cases of dissected innominate artery is to establish CPB via central cannulation of the ascending aorta and provide ACP using flexible, balloon-tipped catheters introduced into the ostia of the supraaortic vessels. ${ }^{18}$ Alternatively, in these cases, we resort to ACP via the axillary artery, or deep hypothermia and RCP.

The debate between unilateral and bilateral cerebral perfusion as the optimal cerebral protection strategy is ongoing. A recent study showed no difference in stroke rate when unilateral or bilateral $\mathrm{ACP}$ was used for cerebral protection during circulatory arrest. ${ }^{19}$ Similarly, the optimal temperature during circulatory arrest and ACP remains to be definitively ascertained. A full discussion of the relative merits of these approaches is beyond the scope of the data presented in this study. However, our data clearly show that in patients with short circulatory arrest times, unilateral cerebral perfusion is safe, even under moderate hypothermic conditions. We only use bilateral cerebral perfusion if there is lateral asymmetry on the EEG and/or $>50 \%$ decrease in cerebral oximetry by near-infrared spectroscopy monitoring. In this series, we did not use bilateral ACP; however, in other cases where longer circulatory arrest periods may be anticipated (eg, acute aortic dissection repair and total aortic arch replacement) bilateral ACP can be established by placement of a balloon-tipped catheter directly into the ostium of the left common carotid artery. It should be noted that we do not aim for EEG silence, and see slow but persistent EEG activity bilaterally throughout the entire course of moderate hypothermic circulatory arrest. Currently, we are in the process of analyzing the effect of ACP under moderate hypothermic conditions on cerebral activity as measured by EEG. Detailed analysis of these data along with more robust neurologic testing will be important in further validating this approach.

A limitation of our study is that only patients undergoing elective hemiarch replacement and short circulatory arrest times were included in our study. Traditional practice at our institution has been to perform transverse hemiarch reconstruction under systemic deep hypothermic 
circulatory arrest with RCP and only recently have some surgeons adopted the strategy of moderate hypothermia and $\mathrm{ACP}^{6}$ In the future, we anticipate investigating the use of this technique for all circulatory arrest cases, including total arch reconstructions. In addition to several previously published approaches to establish ACP during circulatory arrest, we present a safe, alternate technique for cerebral perfusion.

\section{Conflict of Interest Statement}

Authors have nothing to disclose with regard to commercial support.

\section{References}

1. Stein LH, Elefteriades JA. Protecting the brain during aortic surgery: an enduring debate with unanswered questions. J Cardiothorac Vasc Anesth. 2010;24:316-21.

2. Milewski RK, Pacini D, Moser GW, Moeller P, Cowie D, Szeto WY, et al Retrograde and antegrade cerebral perfusion: results in short elective arch reconstructive times. Ann Thorac Surg. 2010;89:1448-57.

3. Pacini D, Pantaleo A, Marco LD, Leone A, Barberio G, Murana G, et al. Visceral organ protection in aortic arch surgery: safety of moderate hypothermia. Eur J Cardiothorac Surg. 2013;46:438-43.

4. Leshnower BG, Myung RJ, Chen EP. Aortic arch surgery using moderate hypothermia and unilateral selective antegrade cerebral perfusion. Ann Cardiothorac Surg. 2013;2:288-95.

5. Tsai JY, Pan W, Lemaire SA, Pisklak P, Lee VV, Bracey AW, et al. Moderate hypothermia during aortic arch surgery is associated with reduced risk of early mortality. J Thorac Cardiovasc Surg. 2013;146:662-7.

6. Vallabhajosyula P, Jassar AS, Menon RS, Komlo C, Gutsche J, Desai ND, et al. Moderate versus deep hypothermic circulatory arrest for elective aortic transverse hemiarch reconstruction. Ann Thorac Surg. 2015;99:1511-7.

7. Schachner T, Nagiller J, Zimmer A, Laufer G, Bonatti J. Technical problems and complications of axillary artery cannulation. Eur J Cardiothorac Surg. 2005;27: 634-7.

8. Anastasiadis K, Antonitsis P, Papakonstantinou C. Innominate artery cannulation. Multimed Man Cardiothorac Surg. 2008;2008. mmets 2008 003418 .
9. Eusanio MD, Petridis FD, Folesani G, Berretta P, Zardin D, Bartolomeo RD Axillary and innominate artery cannulation during surgery of the thoracic aorta: a comparative study. J Cardiovasc Surg. 2013;54:1-7.

10. Hokenek AF, Kinoglu B, Gursoy M, Sirin G, Gulcan F. Direct innominate artery cannulation in surgery for annuloaortic ectasia. J Card Surg. 2013;28:550-3.

11. Preventza O, Bakaeen FG, Stephens EH, Trocciola SM, de la Cruz KI, Coselli JS Innominate artery cannulation: an alternative to femoral or axillary cannulation for arterial inflow in proximal aortic surgery. J Thorac Cardiovasc Surg. 2013; 145(3 Suppl):S191-6.

12. Ji S, Yang J, Ye X, Wang X. Brain protection by using innominate artery cannulation during aortic arch surgery. Ann Thorac Surg. 2008;86:1030-2.

13. Garg V, Tsirigotis DN, Dickson J, Dalamagas C, Latter DA, Verma S, et al. Direct innominate artery cannulation for selective antegrade cerebral perfusion during deep hypothermic circulatory arrest in aortic surgery. J Thorac Cardiovasc Surg. 2014;148:2920-4.

14. Di Eusanio M, Quarti A, Pierri MD, Di Eusanio G. Cannulation of the brachiocephalic trunk during surgery of the thoracic aorta: a simplified technique for antegrade cerebral perfusion. Eur J Cardiothorac Surg. 2004;26: 831-3.

15. Preventza O, Garcia A, Tuluca A, Henry M, Cooley DA, Simpson K, et al. Innominate artery cannulation for proximal aortic surgery: outcomes and neurological events in 263 patients. Eur J Cardiothorac Surg. 2015;48: 937-42.

16. Urbanski PP, Lenos A, Bougioukakis P, Neophytou I, Zacher M, Diegeler A. Mild-to-moderate hypothermia in aortic arch surgery using circulatory arrest: a change of paradigm? Eur J Cardiothorac Surg. 2012;41:185-91.

17. Augoustides JG, Desai ND, Szeto WY, Bavaria JE. Innominate artery cannulation: the Toronto technique for antegrade cerebral perfusion in aortic arch reconstruction-a clinical trial opportunity for the International Aortic Arch Surgery Study Group. J Thorac Cardiovasc Surg. 2014;148: 2924-6.

18. Frederick JR, Yang E, Trubelja A, Desai ND, Szeto WY, Pochettino A, et al. Ascending aortic cannulation in acute type a dissection repair. Ann Thorac Surg. 2013;95:1808-11.

19. Preventza O, Simpson KH, Cooley DA, Cornwell L, Bakaeen FG, Omer S, et al. Unilateral versus bilateral cerebral perfusion for acute type A aortic dissection. Ann Thorac Surg. 2015;99:80-7.

Key Words: aortic arch, cerebral perfusion, moderate hypothermic circulatory arrest 